\title{
A influência da posição acadêmica sobre condutas de saúde em universitários
}

\author{
Marcos Cezar Pomini*; Danielle Bordin**; Nemre Adas Saliba***; Suzely Adas Saliba Moimaz***; \\ Cristina Berger Fadel****
}

* Estudante de graduação em Odontologia, Universidade Estadual de Ponta Grossa

** Doutoranda em Odontologia Preventiva e Social, Faculdade de Odontologia de Araçatuba, UNESP, Professora Colaboradora, Departamento de Enfermagem e Saúde Pública, Universidade Estadual de Ponta Grossa

*** Doutora em Odontologia Preventiva e Social, Faculdade de Odontologia de Araçatuba, UNESP

**** Doutora em Odontologia Preventiva e Social, Faculdade de Odontologia de Araçatuba, UNESP, Professor Adjunto, Departamento de Odontologia, Universidade Estadual de Ponta Grossa

Recebido em 15/02/2017. Aprovado em 02/07/2017.

\section{RESUMO}

Poucos estudos têm examinado as condutas de saúde da população universitária em sua totalidade. O objetivo deste estudo foi avaliar a influência da posição acadêmica, considerando estudantes de graduação e pós-graduação, sobre a aquisição de condutas de saúde. A amostra foi composta por 223 graduandos e 67 pós-graduandos, ambos concluintes dos cursos de Odontologia, Farmácia, Medicina, Educação Física, Enfermagem e Ciências Biológicas de uma universidade pública da região Sul do Brasil. Utilizou-se um instrumento para a coleta de dados demográficos e o questionário autoaplicável National College Health Risk Behavior Survey (NCHRBS), para a avaliação das dimensões segurança no trânsito e violência, consumo de tabaco e álcool, comportamento sexual e alimentação, atividade física e peso. Observou-se que a posição acadêmica não exerceu papel significativo na aquisição de condutas inadequadas, estando estas mais relacionadas com o perfil demográfico dos indivíduos. Estudantes mais jovens, do gênero masculino e solteiros apresentaram maior prevalência de hábitos considerados prejudiciais à saúde, principalmente relacionados ao consumo de bebida alcoólica e ao envolvimento em agressão física. Estudantes mulheres apresentaram aquisição significativa de métodos inapropriados para o controle do peso corporal. Os resultados encontrados refletem a importância da efetivação de políticas que visem à intervenção e prevenção de comportamentos prejudiciais à saúde entre universitários, considerando-se o universo de graduandos e pós-graduandos.

Descritores: Comportamentos Saudáveis. Risco. Estudantes. Educação Superior. 


\section{INTRODUÇÃ̃O}

As condutas de saúde e os malefícios da aquisição de condutas inadequadas por acadêmicos universitários da graduação têm sido bastante explorados pela literatura nacional e internacional ${ }^{1-6}$. Em geral, estudos apontam alta prevalência de tabagismo, consumo de bebidas alcoólicas e uso de drogas entre universitários ${ }^{1,2}$, apesar de resultados mostrarem redução na prevalência de exposição a essas condutas de risco em populações jovens ${ }^{7,8}$. $\mathrm{O}$ baixo nível de atividades físicas e a alimentação inadequada, com consumo insuficiente de frutas e de hortaliças e rica em alimentos gordurosos e doces, também são identificados na população acadêmica ${ }^{9-11}$.

Pesquisas demonstram ainda que a exposição às principais condutas de risco está associada ao estresse psicossocial em adultos ${ }^{12,13} \mathrm{e}$ que a relação entre o fenômeno estresse e a vivência do ensino superior está bem estabelecida. Esses dados sugerem que o estresse e a ansiedade sejam responsáveis por influenciar os acadêmicos para novos comportamentos e relações sociais, transformando os novos hábitos em "válvulas de escape" $" 13,14$. Dentre as principais fontes geradoras de estresse para acadêmicos, encontram-se, por exemplo, a sobrecarga curricular, as características próprias do ambiente institucional e a perda da maneabilidade sobre o tempo e o lazer ${ }^{15}$.

Frequentemente o período universitário vem sendo relacionado ao risco ampliado para aquisição de hábitos prejudiciais a saúde ${ }^{16,17}$. No entanto, a posição ocupada pelo acadêmico universitário dentro de seu processo de formação e qualificação profissional (graduação ou pós-graduação) e seus efeitos na alteração de condutas de saúde permanece pouco explorada em estudos conduzidos no Brasil.

Os hábitos adquiridos pelos estudantes durante o ensino superior podem permanecer durante a vida adulta ${ }^{18}$, assim, o conhecimento dos fatores que se relacionam com a adoção de condutas de saúde entre universitários é fundamental para a inserção de medidas de intervenção adequadas visando à aplicação de estratégias preventivas satisfatórias ${ }^{17,19}$. Nesse sentido, a exploração da relação entre a posição do acadêmico no ensino superior e a manifestação de comportamentos em saúde pode direcionar ações institucionais aos grupos mais vulneráveis a condutas consideradas negativas ou indesejáveis, auxiliando assim na caracterização de um grupo populacional, nas técnicas de intervenção necessárias e satisfatórias e na avaliação da eficácia das mesmas.

Por estas considerações e pela ciência de que o objeto exposto deve estar presente na agenda do gestor em educação na saúde, o objetivo do presente estudo foi investigar a influência da variável posição acadêmica, considerando estudantes de graduação e pós-graduação, sobre condutas de saúde de universitários.

Como hipótese deste estudo tem-se que existam diferenças em relação às condutas de saúde de concluintes graduandos e pós-graduandos, com pior desempenho para pós-graduandos. Além disso, a literatura tem apontado que o desenvolvimento científico brasileiro se dá à custa de grande estresse e desgaste emocional por parte dos pesquisadores, dentre eles os alunos de pós-graduação, especialmente no que tange à constante demanda por aumento no volume de produção bibliográfica ${ }^{20,21}$. Assim, supõese que as exigências vivenciadas pelo acadêmico de pós-graduação possam influenciar negativamente os seus comportamentos de saúde.

\section{METODOLOGIA}

Trata-se de um estudo transversal, quantitativo de caráter exploratório. $\mathrm{O}$ estudo foi aprovado pelo Comitê de Ética em Pesquisas com 
seres humanos da instituição (parecer $\mathrm{n}^{\mathrm{o}}$ 572.627/2014), respeitando os ditames da resolução 466/12 do Conselho Nacional de Saúde.

Amostra por conveniência foi formada pela totalidade de acadêmicos concluintes graduandos $(\mathrm{n}=279)$ e pós-graduandos stricto sensu $(\mathrm{n}=95)$ dos cursos presenciais de Ciências Biológicas e da Saúde regularmente ofertados por uma universidade pública do Sul do Brasil, a saber: Odontologia, Enfermagem, Farmácia, Medicina, Bacharelado e Licenciatura em Educação Física e Bacharelado e Licenciatura em Ciências Biológicas. Todos os cursos de graduação são em tempo integral, com duração média de cinco anos. Já os cursos de pós-graduação têm em média dois anos (mestrado) e quatro anos (doutorado). Os indivíduos foram identificados por meio de listagem fornecida pela instituição de ensino e o ano base para a consulta foi 2015 (agosto a dezembro). O critério de inclusão foi o acadêmico estar matriculado no último período letivo de um dos cursos citados, independente de variáveis sociais ou demográficas.

Para a coleta de informações utilizou-se um instrumento de caracterização da amostra contendo as variáveis demográficas idade, gênero, estado civil; a variável dependente posição acadêmica (graduação ou pós-graduação), e o questionário National College Health Risk Behavior Survey (NCHRBS) desenvolvido pelo Centers for Disease Control and Prevention (CDC) dos Estados Unidos em 1995, traduzido para o português e validado para utilização com universitários brasileiros ${ }^{22}$. Este questionário é composto por 50 questões de múltipla escolha que visam captar informações sobre hábitos de saúde considerados prejudicais. O questionário está dividido em cinco dimensões, sendo nove questões relacionadas à segurança no trânsito, quatro a comportamentos relacionados à violência, 15 ao consumo de drogas lícitas e ilícitas, oito ao comportamento sexual e 14 aos hábitos alimentares, percepção do peso e prática de atividade física. Detalhes sobre o processo de validação e ponderação estão disponíveis no estudo de Franca e Colares ${ }^{22}$. Os instrumentos eram autoaplicáveis e foram submetidos à préteste com acadêmicos não-concluintes da mesma instituição, de ambas as posições acadêmicas descritas.

Os dados foram coletados por pesquisador calibrado, de forma presencial, individual e em momento considerado propício pelos entrevistados que concordaram em participar da pesquisa. Previamente foram explicitados os propósitos do estudo, a voluntariedade de participação e a garantia de sigilo durante todas as etapas da pesquisa.

Inicialmente, análises descritivas da variável dependente e dos dados do NCHRBS foram efetuadas por distribuição de frequências, enquanto que para as análises bivariadas recorreuse à aplicação do teste de qui-quadrado ou o teste exato de Fisher, quando as condições para a utilização do primeiro não foram atendidas. Em seguida, somente os dados (condutas de saúde) que apontaram diferença significativa entre os grupos de estudo foram novamente tratados e analisados, a fim de minimizar vieses de confundimento com as variáveis demográficas e atribuir associação verdadeira com o fato do acadêmico estar cursando a graduação ou a pós-graduação.

\section{RESULTADOS}

Participaram do estudo 290 universitários concluintes, sendo 223 de graduação e 67 de pósgraduação. Dentre os acadêmicos de graduação, constatou-se a participação de 10 estudantes de Bacharelado em Ciências Biológicas, 26 de Licenciatura em Ciências Biológicas, 37 de 
Medicina, 38 de Odontologia, 35 de Farmácia, 32 de Enfermagem, 23 de Licenciatura em Educação Física e 22 de Bacharelado em Educação Física. Já entre os pós-graduandos, 12 eram alunos do Mestrado em Ciências Biomédicas, 17 em Ciências Biológicas, 10 em Ciências Farmacêuticas, 16 em Odontologia e 12 do Doutorado em Odontologia. Dentre os acadêmicos de graduação, a média etária foi de $23 \pm 1,44$ anos, solteiros (95\%) e mulheres $(80 \%)$ em sua maioria. Os acadêmicos pós-graduandos tinham em média $26 \pm 1,90$ anos, sendo $77,6 \%$ mulheres e $22,4 \%$ homens, solteiros em sua maioria ( $81 \%)$.

A tabela 1 apresenta as condutas de saúde que apresentaram significância estatística quando associadas aos grupos acadêmicos analisados. Nenhuma outra dimensão ou conduta, independente da dimensão a que pertence, mostrou condição similar.

Tabela 1. Distribuição das dimensões e condutas do instrumento NCHRBS com significância estatística, segundo posição acadêmica

\begin{tabular}{|c|c|c|c|}
\hline Dimensão/Conduta & $\begin{array}{c}\text { Graduação } \\
\text { n=223 } \\
\text { n(\%) }\end{array}$ & $\begin{array}{c}\text { Pós-Graduação } \\
\text { n= } 67 \\
\text { n(\%) }\end{array}$ & $p$ valor \\
\hline $\begin{array}{l}\text { Segurança no trânsito e violência } \\
\text { Envolver-se em agressão física no último ano }\end{array}$ & $19(8,5)$ & $1(1,5)$ & $0,032 * *$ \\
\hline $\begin{array}{l}\text { Consumo de tabaco e álcool } \\
\text { Consumo de álcool no último mês }\end{array}$ & $159(71,3)$ & $56(83,6)$ & $0,044 *$ \\
\hline $\begin{array}{l}\text { Comportamento sexual } \\
\text { Realizado teste para verificar HIV }\end{array}$ & $77(34,5)$ & $38(56,7)$ & $0,001 *$ \\
\hline $\begin{array}{l}\text { Alimentação, atividade física e peso } \\
\text { Realizar dieta para emagrecer ou manter o peso }\end{array}$ & $70(31,4)$ & $32(47,8)$ & $0,013 *$ \\
\hline
\end{tabular}

A tabela 2 mostra que a condição declarada de estar solteiro foi significativa para a variável 'consumo de bebida alcoólica no último mês', não influenciando as demais condutas de saúde analisadas. Já a variável demográfica gênero foi significativa para a prevalência de homens com envolvimento em agressão física no último ano e de mulheres realizando dieta para emagrecer ou perder peso.

Após a análise por gênero e estado civil, verificou-se que a relação das variáveis encontradas no primeiro teste com a variável de interesse posição acadêmica, que expõe a relação entre graduandos e pós-graduandos, permaneceu associada somente à dimensão comportamento sexual, com a conduta 'ter realizado teste para verificar a presença de HIV'. As demais variáveis tiveram relação com gênero e idade. Ao avaliar esta conduta de saúde e o fator idade verificou-se diferença significativa somente para os entrevistados com 27 a 29 anos (tabela 3). Vale destacar que a totalidade dos acadêmicos graduandos esteve concentrada na faixa etária de 21 a 26 anos, enquanto os pós-graduandos dividiram-se nas faixas acima de 24 anos. Deste modo, estes resultados devem ser observados com cautela.

Na tabela 3 pode-se verificar ainda diferença significativa para as variáveis relacionadas ao consumo de tabaco e álcool e alimentação, atividade física e peso. 
Tabela 2. Distribuição das dimensões e condutas do instrumento NCHRBS com significância estatística, segundo estado civil e gênero

\begin{tabular}{|c|c|c|c|c|c|c|}
\hline Dimensão/Conduta & $\begin{array}{c}\text { Casado } \\
\text { n=27 } \\
\text { n }(\%)\end{array}$ & $\begin{array}{c}\text { Solteiro } \\
\mathrm{n}=\mathbf{2 6 3} \\
\mathrm{n}(\%)\end{array}$ & p valor & $\begin{array}{c}\text { Homem } \\
\text { n=59 } \\
\text { n }(\%)\end{array}$ & $\begin{array}{c}\text { Mulher } \\
\text { n=231 } \\
\text { n }(\%)\end{array}$ & p valor \\
\hline $\begin{array}{l}\text { Segurança no trânsito e violência } \\
\text { Envolver-se em agressão física no } \\
\text { último ano }\end{array}$ & $0(0)$ & $20(7,6)$ & $0,131 * *$ & $19(32,2)$ & $1(2,3)$ & $0,000 * *$ \\
\hline $\begin{array}{l}\text { Consumo de tabaco e álcool } \\
\text { Consumo de álcool no último mês }\end{array}$ & $15(55,5)$ & $200(76)$ & $0,020 *$ & $39(66,1)$ & $176(76,2)$ & $0,114^{*}$ \\
\hline $\begin{array}{l}\text { Comportamento sexual } \\
\text { Realizado teste para verificar HIV }\end{array}$ & $09(33,3)$ & $106(40,3)$ & $0,314 *$ & $18(30,5)$ & $97(42)$ & $0,481 *$ \\
\hline $\begin{array}{l}\text { Alimentação, atividade física e pes } \\
\text { Realizar dieta para emagrecer ou } \\
\text { manter o peso }\end{array}$ & $07(25,9)$ & $95(36,1)$ & $0,291 *$ & $13(22)$ & $89(38,5)$ & $0,018^{*}$ \\
\hline
\end{tabular}

Tabela 3. Distribuição das dimensões e condutas do instrumento NCHRBS com significância estatística, segundo a idade

\begin{tabular}{|c|c|c|c|c|c|}
\hline Dimensão/Conduta & $\begin{array}{c}21-23 \\
n=150 \\
n(\%)\end{array}$ & $\begin{array}{c}24-26 \\
n=115 \\
n(\%)\end{array}$ & $\begin{array}{c}27-29 \\
n=18 \\
n(\%)\end{array}$ & $\begin{array}{c}>29 \\
n=07 \\
n(\%)\end{array}$ & p valor \\
\hline $\begin{array}{l}\text { Segurança no trânsito e violência } \\
\text { Envolver-se em agressão física no último ano }\end{array}$ & $15(10)$ & $04(3,5)$ & $0(0)$ & $01(14,3)$ & $* 0,099$ \\
\hline $\begin{array}{l}\text { Consumo de tabaco e álcool } \\
\text { Consumo de álcool no último mês }\end{array}$ & $123(82)$ & $71(61,7)$ & $17(94,4)$ & $04(57,1)$ & $* 0,000$ \\
\hline $\begin{array}{l}\text { Comportamento sexual } \\
\text { Realizado teste para verificar HIV }\end{array}$ & $30(20)$ & $62(53,9)$ & $17(94,4)$ & $6(85,7)$ & $* 0,000$ \\
\hline $\begin{array}{l}\text { Alimentação, atividade física e peso } \\
\text { Realizar dieta para emagrecer ou manter o peso }\end{array}$ & $52(34,7)$ & $30(26,1)$ & $16(88,9)$ & $04(57,1)$ & $* 0,000$ \\
\hline
\end{tabular}

\section{DISCUSSÃO}

$\mathrm{Na}$ maioria das condutas de saúde investigadas não foi verificada diferença significativa entre as posições acadêmicas. Nas variáveis que demonstraram relação, a influência esteve dissolvida no contexto das variáveis demográficas. Deste modo, pode-se inferir que a posição acadêmica teve pouca influência sobre as condutas em saúde, estando estas, mais frequentemente relacionadas com o gênero, estado 
civil ou idade. De fato, a literatura tem demonstrado que sexo, idade, etnia, cor, renda e escolaridade influenciam consideravelmente na aquisição de condutas prejudiciais, assim como na procura de atendimentos nas redes públicas e particulares de saúde ${ }^{23-26}$.

Cotrim et $a l .{ }^{27}$, em uma avaliação dos comportamentos de saúde em estudantes, demonstraram que homens estão mais associados a comportamentos prejudiciais à saúde, especialmente relacionados à conduta no trânsito, violência física e porte de arma $^{27}$, assemelhandose aos resultados do presente estudo, no qual estudantes do gênero masculino apresentaram prevalência significativa de envolvimento em agresssão física. Universitários do gênero feminino apresentaram maior prevalência apenas para a conduta 'uso de estratégias não saudáveis para controle do peso corporal', corroborando dados mundiais $^{28,29}$.

Estudos também apontam para a influência do estado civil e da idade nas práticas comportamentais relacionadas à saúde ${ }^{30-32}$. Evidências científicas demonstram que indivíduos solteiros tendem a consumir bebida alcoólica em maior quantidade quando comparados a $\operatorname{casados}^{30,31}$; que jovens são mais suscetíveis à aquisição de práticas adversas à saúde ${ }^{32}$; e que estes riscos tendem a permanecer durante a vida, aumentando a taxa de mortalidade ${ }^{2,33,34}$. O autorrelato dos estudantes no presente trabalho reflete o atual cenário de jovens brasileiros, onde a aquisição de condutas prejudiciais à saúde está em constante aumento, visando ao escape da realidade e ao suprimento das exigências sociais, especialmente relacionado à adoção de medidas de risco sobre a estética corporal e o consumo de álcool ${ }^{35,36}$.

Outra indicação dos resultados traz à tona questões relativas ao nível de escolaridade. Julgava-se que a vivência ampliada de um cenário de pós-graduação nas áreas da saúde, repleta de discussões de excelência e de alto nível de criticidade, pudesse influenciar condutas de saúde. Estudos expõem relação de proximidade entre o nível de escolaridade alcançado por um indivíduo e a adoção de comportamentos saudáveis ${ }^{23,24,25}$. As maiores taxas encontradas nesta população podem ser explicadas pelo fato de que estes indivíduos tendem a ter uma compreensão maior dos efeitos do tratamento sobre as doenças e do risco de aquisição de condutas prejudiciais à saúde, bem como maior discernimento quanto às diferentes modalidades e especialidades que regem a aquisição e manutenção da saúde. Em um estudo observacional, Silva et al. ${ }^{24}$ demonstraram que grupos de renda e escolaridade mais elevadas e mulheres apresentam maior procura por serviços de saúde. Pode-se acomodar nessa afirmação a busca ratificada no presente estudo pela realização de teste diagnóstico para verificar a presença de HIV.

No estudo em questão e considerando-se em especial o grupo de pós-graduandos, sugere-se a presença de uma cultura de valorização dos aspectos que norteiam o campo científico e profissional. Severino ${ }^{37}$ evidencia que a condução da vida do pós-graduando deve ser impregnada pela incorporação de espírito investigativo dedicado à construção do conhecimento e inserida em contexto problematizador. Além disso, os ambientes institucionais de pós-graduação acabam fornecendo somente subsídios de infraestrutura e tecnológicos para o desenvolvimento satisfatório de pesquisas, gerando uma espécie de alheamento de questões voltadas à condição da integralidade humana, incluindo a adoção de condutas saudáveis. A falta de investimento em cenários de 
discussão e socialização acerca de fatores que afetam a saúde e a qualidade de vida de pósgraduandos ajuda a explicar a similaridade encontrada entre as condutas de saúde investigadas e a posição acadêmica.

Os resultados alcançados mostram que as políticas de intervenção relacionadas a condutas de saúde entre os acadêmicos analisados podem seguir uma orientação única por parte de gestores educacionais, uma vez que as diferenças existentes entre pós-graduandos e graduandos foram muito mais de caráter intrínseco às condições demográficas do que ao grau de formação. No entanto, como os cursos de graduação em saúde assumem com frequência a missão de promover cursos e programas de saúde, visualiza-se a necessidade de desenvolver nos cursos de pósgraduação momentos onde possam ser discutidos aspetos relevantes de comportamento e condutas de saúde.

Estudos sobre a presença e a efetividade de políticas estratégicas para aquisição de comportamentos saudáveis junto à população universitária, especialmente na pós-graduação, podem contribuir para reduzir os comportamentos de risco junto às comunidades acadêmicas e direcionar estratégias efetivas para controle e prevenção dos mesmos. Exemplo de estratégia efetiva foi descrito no estudo de Deliens et al. ${ }^{38}$ realizado com estudantes universitários. Os autores constataram que investir na redução do preço de frutas e no aumento, em igual proporção, do valor de batatas fritas reduz o consumo de alimentos não saudáveis e aumenta o de saudáveis, auxiliando o processo de mudança de comportamento.

O subsídio à adoção de práticas saudáveis traz impactos individuais, coletivos, sociais e econômicos bastante significativos. Estudos nacionais e internacionais apontam que pessoas que aderem a comportamentos saudáveis apresentam maior longevidade ${ }^{33,39}$, redução da vulnerabilidade ao adoecer ${ }^{25,33,39}$, e da morbimortalidade geral ${ }^{25,26,40}$, além da diminuição do impacto financeiro sobre o sistema de saúde e de efeitos adversos em sua qualidade de vida ${ }^{25,41}$.

Torna-se importante ressaltar que a representatividade da amostra neste estudo é uma questão que deve ser avaliada com cautela. Ainda que a mesma tenha englobado acadêmicos de diversos cursos da área de Ciências Biológicas e da Saúde, pode não ser representativa do universo de graduandos e pós-graduandos de diferentes instituições de ensino. Deste modo, e para efeito de validação externa, sugere-se que estudos similares sejam realizados em outros núcleos educacionais, envolvendo áreas ampliadas da formação acadêmica.

Ainda, para fins de análise e de alcance do objetivo proposto, considerou-se somente a presença ou a ausência das condutas de saúde avaliadas, independente de suas nuances de ocorrência. Esse fato sugere a importância de investigação dessas condutas também em termos de frequência e duração de exposição.

\section{CONCLUSÃO}

Conclui-se que a posição acadêmica, isoladamente, apresentou pouca influência sobre as condutas de saúde, visto que em todas as condutas analisadas verificou-se também influência de variáveis demográficas. A hipótese deste estudo não foi confirmada. Deste modo, os dados encontrados expõem a necessidade de atuação de gestores institucionais, de forma comum e integrada, junto a estudantes de graduação e pós-graduação, por meio da efetivação de políticas que visem à prevenção e 
intervenção de comportamentos de saúde.

\section{ABSTRACT \\ The influence of the university level on health conducts in university students}

Few studies have examined healthy behaviors of the university population in its entirety. The aim of this study was to evaluate the influence of the academic position, considering undergraduate and graduate students, on the acquisition of healthy behaviors. The sample consisted of 223 undergraduate and 67 postgraduate students of the Life and Health Sciences area (Dentistry, Pharmacy, Medicine, Physical Education, Nursing and Biological Sciences courses) of a public university in southern Brazil. An instrument for the collection of demographic data and the National College Health Risk Behavior Survey (NCHRBS) was used to assess four dimensions: security in traffic and violence, tobacco and alcohol consumption, sexual behavior and nutrition, and physical activity and weight. It was observed that the academic position did not play a significant role in the acquisition of risky habits, being this acquisition more related to the demographic profile of the individuals. Younger, male and unmarried students had a higher prevalence of habits considered harmful to health, mainly related to alcohol consumption and involvement in physical aggression. Female students presented statistical significant difference for using inappropriate methods for body weight control. The results reflect the importance of effective policies, which aim at intervention and prevention of risk behaviors among the college population, considering the undergraduate and graduate students.

Descriptors: Health Behavior. Risk Behavior. Students. Education, Higher.

\section{REFERÊNCIAS}

1. Andrade AG, Duarte PCAV, Barroso LP, Nishimura R, Alberghini DG, Oliveira LG.
Use of alcohol and other drugs among Brazilian college students: effects of gender and age. Rev Bras Psiquiatr. 2012;34:294305.

2. Pedrelli P, Bitran S, Shyu I, Baer L, Guidi J, Tucker DD, et al. Compulsive alcohol use and other high-risk behaviors among college students. Am J Addict. 2011;20(1):14-20.

3. Chavez KAP, O’Brien B, Pillon SC. Drugs use and risk behavior in a university community. Rev Latino-Am Enfermagem. 2005;13:1194-200.

4. Ramis TR, Mielke GI, Habeyche EC, Oliz MM, Azevedo MR, Hallal PC. Tabagismo e consumo de álcool em estudantes universitários: prevalência e fatores associados. Rev Bras Epidemiol. 2012;12(2):376-85.

5. Andrade AG, Duarte PCAV, Barroso LP, Nishimura R, Alberghini DG, Oliveira LG. Use of alcohol and other drugs among Brazilian college students: effects of gender and age. Rev Bras Psiquiatr. 2012;34:294305.

6. Cecchini M, Sassi F, Lauer JA, Lee YY, Guajardo-Barron V, Chisholm D. Tackling of unhealthy diets, physical inactivity, and obesity: health effects and cost-effectiveness. Lancet. 2010;376(9754):1775-84.

7. Eaton DK, Kann L, Kinchen S, Shanklin S, Ross J, Hawkins J, et al. Youth risk behavior surveillance - United States, 2009. MMWR Surveill Summ. 2010;59(5):1-142.

8. Niclasen B, Schnohr C. Has the curve been broken? Trends between 1994 and 2006 in smoking and alcohol use among Greenlandic school children. Int J Circumpolar Health. 2008;67:299-307.

9. Racette SB, Deusinger SS, Strube MJ, Highstein GR, Deusinger RH. Weight 
changes, exercise, and dietary patterns during freshman and sophomore years of College. $\mathrm{J}$ Am Coll Health. 2005;53(6):245-51.

10. Vargas-Zárate M, Becerra-Bulla F, PrietoSuárez E. Evaluación de la ingesta dietética en estudiantes universitarios. Rev Salud Pública. 2010;12(1):116-25.

11. Sousa TF. Inatividade física em universitários brasileiros: uma revisão sistemática. Rev Bras Ciênc Saúde. 2011;9(29):47-55.

12. Harling M, Strehmel P, Schablon A, Nienhaus A. Psychosocial stress, demoralization and the consumption of tobacco, alcohol and medical drugs by veterinarians. J Occup Medical Toxicology. 2009;4:4.

13. Hudd SS, Dumlao J, Erdmann-Sager, Murray D, Phan E, Soukas N. Stress at college: effects oh health habits, health status and self-esteem. Coll Stud J. 2000;34(2):217.

14. Vieira VCR, Priore SE, Ribeiro SMR, Franceschini SCC, Almeira LP. Perfil socioeconômico, nutricional e de saúde de adolescentes recém-ingressos em uma universidade pública brasileira. Rev Nutr. 2002;15(3):273-82.

15. Loureiro E, Mcintyre T, Mota-Cardoso R, Ferreira MA. A relação entre o stress e os estilos de vida nos estudantes de medicina da Faculdade de Medicina do Porto. Acta Med Port. 2008;21(3):209-14.

16. Park CL, Grant C. Determinant of positive and negative consequences of alcohol consumption in college students: alcohol use, gender e psychological characteristics. Addict Behav. 2005; 30(4):755-65.

17. Peuker AC, Fogaça J, Bizarro L. Expectativas e beber problemático entre universitários. Psic Teor e Pesq. 2006;22(2):193-200.

18. Marcondelli P, Costa THM, Schmitz BAS. Physical activity level and food intake habits of university students from 3 to 5 semester in the health area. Rev Nutr. 2008;21(1):39-47.

19. Matos APS, Sousa-Albuquerque CM. Estilo de vida, percepção de saúde e estado de saúde em estudantes universitários portugueses: influência da área de formação. Int J Clin Health Psychol. 2006;6(3):647-63.

20. Malagris LEN, Suassuna ATR, Bezerra DV, Hirata HP, Monteiro JLF, Silva LR, et al. Níveis de estresse e características sociobiográficas de alunos de pós-graduação. Psicol Rev. 2009;15(2):184-203.

21. Faro A. Estresse e estressores na PósGraduação: estudo com mestrandos e doutorandos no Brasil. Psic Teor e Pesq. 2013;29(1):51-60.

22. Franca C, Colares V. Validação do National College Health Risk Behavior Survey para a utilização com universitários brasileiros. Ciênc Saúde Coletiva. 2010; 15(1)1209-15.

23. Neri M, Soares W. Social inequality and health in Brazil. Cad Saúde Pública. 2002;18:77-87.

24. Silva ZP, Ribeiro MCSA, Barata RB, Almeida MF. Perfil sóciodemográfico e padrão de utilização dos serviços de saúde do Sistema Único de Saúde (SUS), 2003-2008. Ciênc Saúde Coletiva. 2011;16(9):3807-16.

25. Malta DC, Silva-Junior JB. O Plano de Ações Estratégicas para o Enfrentamento das Doenças Crônicas Não Transmissíveis no Brasil e a definição das metas globais para o enfrentamento dessas doenças até 2025: uma revisão. Epidemiol Serv Saude. 2013;22 (1):151-64.

26. Szwarcwald CL, Sousa Júnior PRB, Damascena GN, Almeida WS, Malta DC, Stoppa SR, et al. Recommendations and practice of healthy behaviors among patients with diagnosis and diabetes in Brazil: National Health Survey (PNS), 2013. Rev Bras Epidemiol. 2015;18(2):132-45.

27. Cotrim BC, Gazal-Carvalho C, Gouveia N. Comportamentos de saúde entre jovens estudantes das redes pública e privada da área metropolitana do Estado de São Paulo. Rev Saud Pub. 2000;34(6): 636- 45.

28. Micali N, Hagberg KW, Petersen I, Treasure JL. The incidence of eating disorders in the UK 2000-2009. Brit Med J. 2013;3(5). 
29. Want, S. Meta-analytic moderators of experimental exposure to media portrayals of women on female appearance satisfaction: Social comparisons as automatic processes. Body Image. 2009;6(4):257-69.

30. Bogart LM, Collins RL, Ellickson PL, Martino SC, Klein DJ. Effects of early and later marriage on women's alcohol use in young adulthood: a prospective analysis. J Stud Alcohol Drugs. 2005;66(6):729-37.

31. Kendler KS, Lönn SL, Salvatore J, Sundquist J, Sundquist K. Effect of marriage on risk for onset of alcohol use disorder: a longitudinal and co-relative analysis in a Swedish national sample. Am J Psychiatry. 2016;173(9):911-8.

32. Currie C, Zanotti C, Morgan A, Currie D, Looze M, Roberts $\mathrm{C}$, et al. Social determinants of health and wellbeing among young people. Health Behaviour in Schoolaged Children (HBSC) study: international report from the 2009/2010 survey. WHO. 2012 [Acesso em 12 ago.2016]. Disponível em: http://www.euro.who.int/data/assets/pdf file/0003/163857/Social-determinants-of-healthand-well-being-among-young-people.pdf.

33. May AM, Struijk EA, Fransen HP, OnlandMoret NC, de Wit GA, Boer JM, et al. The impact of a healthy lifestyle on DisabilityAdjusted Life Years: a prospective cohort study. BMC 2015;13:39.

34. Ford ES, Bergmann MM, Boeing H, Li C, Capewell S. Healthy lifestyle behaviors and all-cause mortality among adults in the United States. Prev Med. 2012; 55(1):23-7.

35. Malta DC, Silva Junior JB. O Plano de Ações Estratégicas para o Enfrentamento das Doenças Crônicas Não Transmissíveis no Brasil e a definição das metas globais para o enfrentamento dessas doenças até 2025: uma revisão. Epidemiol Serv Saúde. 2013; 22(1):151-64.
36. Coqueiro RS, Petroski EL, Pelegrini AB, Rodrigues A. Insatisfação com a imagem corporal: avaliação comparativa da associação com estado nutricional em universitários. Rev Psiquiatr RS. 2008;30(1):31-8.

37. Severino AJ. Pós-graduação e pesquisa: o processo de produção e de sistematização do conhecimento. Rev Diálogo Educ. 2009;9(26):13-27.

38. Deliens T, Deforche B, Annemans L, De Bourdeaudhuij I, Clarys P. Effectiveness of pricing strategies on french fries and fruit purchases among university students: results from an on-campus restaurant experiment. PLoS ONE. 2016; 11(11):e0165298.

39. Heir T, Erikssen J, Sandvik L. Life style and longevity among initially healthy middle-aged men: prospective cohort study. BMC Public Health. 2013;13: 831.

40. Oyebode O, Gordon-Dseagu V, Walker A, Mindell JS. Fruit and vegetable consumption and all-cause, cancer and CVD mortality: analysis of Health Survey for England data. J Epidemiol Community Health. 2014;68(9): 856-62.

41. Scarborough $\mathrm{P}$, Bhatnagar $\mathrm{P}$, Wickramasinghe $\mathrm{KK}$, Allender S, Foster C, Rayner M. The economic burden of ill health due to diet, physical inactivity, smoking, alcohol and obesity in the UK: an update to 2006-07 NHS costs. J Public Health. 2011;33(4):527-35.

Correspondência para:

Profa. Cristina Berger Fadel

e-mail: cbfadel@gmail.com

Rua Dr. Paula Xavier, 909

84010-270 Ponta Grossa/PR 